\title{
Aspects of unconditionality of bases in spaces of compact operators
}

\author{
by James R. Holub (Blacksburg, Va.)
}

\begin{abstract}
E. Tutaj has introduced classes of Schauder bases termed "unconditionallike" (UL) and "unconditional-like*" (UL*) whose intersection is the class of unconditional bases. In view of this association with unconditional bases, it is interesting to note that there exist Banach spaces which have no unconditional basis and yet have a basis of one of these two types (e.g., the space $\mathcal{O}[0,1])$. In the same spirit, we show in this paper that the space of all compact operators on a reflexive Banach space with an unconditional basis has a basis of type UL*, even though it is well-known that this space has no unconditional basis.
\end{abstract}

1. Introduction. In the papers [6] and [7] E. Tutaj has introduced and studied the fundamental properties of two classes of Schauder bases in Banach spaces related closely to unconditional bases and consequently termed "unconditional-like" (UL) and "unconditional-like*" (UL*). In particular, a Schauder basis $\left\{x_{n}\right\}_{n=1}^{\infty}$ for a real Banach space $E$ is said to be of type UL if convergence of $\sum_{n=1}^{\infty} a_{n} x_{n}$ implies convergence of $\sum_{n=1}^{\infty}\left|a_{n}\right| x_{n}$, and of type $\mathrm{UL}^{*}$ if whenever $\sum_{n=1}^{\infty}\left|a_{n}\right| x_{n}$ converges, so does $\sum_{n=1}^{\infty} a_{n} x_{n}$. Since an unconditional basis is one for which convergence of the series $\sum_{n=1}^{\infty} a_{n} x_{n}$ implies the convergence of every rearrangement as well, one can show that a basis is unconditional if and only if it is of type UL and of type UL* (see [6]), and in view of this relationship it is natural to designate bases of either of these two types as "unconditional-like" and to think of each as having some essential aspect of unconditionality even if they are, in fact, conditional. What is interesting is that though a Banach space may not have an unconditional basis, it may still have a basis of type UL or UL*. For example, Tutaj [6] has demonstrated the existence of a basis of type UL in the space $D$ of Lindenstrauss, which is known to have no unconditional basis

1991 Mathematics Subject Classification: Primary 46B15; Secondary 46E40.

Key words and phrases: unconditional basis, unconditional-like basis, tensor product basis, compact operator space. 
[3], and a basis of type $\mathrm{UL}^{*}$ in $\mathcal{C}[0,1]$ (see [7]), another space which has no unconditional basis [2].

In the same spirit we will show in this paper that if $E$ is a reflexive Banach space having an unconditional basis $\left\{x_{n}, x_{n}^{*}\right\}_{n=1}^{\infty}$, then the standard "tensor product" basis $\left\{x_{n}^{*} \otimes x_{m}\right\}$ of one-dimensional operators for the space $K(E)$ of compact operators on $E$ is of type $\mathrm{UL}^{*}$, even though it is known from results of Pełczyński and Kwapień [4] that $K(E)$ has no unconditional basis.

2. Recall that if $E$ is a reflexive Banach space having a Schauder basis $\left\{x_{n}\right\}_{n=1}^{\infty}$ with coefficient functionals $\left\{x_{n}^{*}\right\}_{n=1}^{\infty}$ in $E^{*}$, then $\left\{x_{n}^{*}\right\}_{n=1}^{\infty}$ is a basis for $E^{*}$ and the sequence of one-dimensional operators $\left\{x_{n}^{*} \otimes x_{m}\right\}$ ordered in "blocks" $\left\{B_{k}\right\}_{k=1}^{\infty}$ of $2 k-1$ operators, $k=1,2, \ldots$, as $\left\{B_{1}, \ldots, B_{k}, \ldots\right\}=$ $\left\{x_{1}^{*} \otimes x_{1}, x_{1}^{*} \otimes x_{2}, x_{2}^{*} \otimes x_{2}, x_{2}^{*} \otimes x_{1}, \ldots, x_{1}^{*} \otimes x_{k}, x_{k}^{*} \otimes x_{k-1}, \ldots, x_{k}^{*} \otimes x_{1}, \ldots\right\}$ is a basis for $E^{*} \otimes_{\lambda} E$, the completion of the linear space $E^{*} \otimes E$ of all finitedimensional operators on $E$ in the operator norm, and hence for $K(E)$, which is identified with $E^{*} \otimes_{\lambda} E$ in this case. Gelbaum and Gil de Lamadrid [1] showed that if $\left\{x_{n}, x_{n}^{*}\right\}_{n=1}^{\infty}$ is an unconditional basis in $E$, the tensor product basis $\left\{x_{n}^{*} \otimes x_{m}\right\}$ for $K(E)$ need not be unconditional, even for the case of an orthonormal basis in Hilbert space, a result extended by Pełczyński and Kwapień in the paper referred to above [4]. Our purpose is to prove the following result which shows that, in spite of these negative results concerning the existence of unconditional bases in $K(E)$, any tensor product basis $\left\{x_{n}^{*} \otimes x_{m}\right\}$ is always of type $\mathrm{UL}^{*}$.

THEOREM. If $E$ is a reflexive Banach space and $\left\{x_{n}, x_{n}^{*}\right\}_{n=1}^{\infty}$ an unconditional basis for $E$, the basis $\left\{x_{n}^{*} \otimes x_{m}\right\}$ for $K(E)$ is of type $\mathrm{UL}^{*}$.

Proof. For convenience of notation, we will denote the basis $\left\{x_{n}^{*} \otimes x_{m}\right\}$ for $K(E)$ (in the order described above) as a sequence $\left\{T_{j}\right\}_{j=1}^{\infty}$ of onedimensional operators of the form $x_{n}^{*} \otimes x_{m}$; i.e., $\left\{T_{j}\right\}_{j=1}^{\infty}=\left\{x_{1}^{*} \otimes x_{1}, x_{2}^{*} \otimes\right.$ $\left.x_{1}, x_{2}^{*} \otimes x_{2}, x_{1}^{*} \otimes x_{2}, \ldots\right\}=\left\{B_{1}, \ldots, B_{k} \ldots\right\}$, as we described earlier. Correspondingly, the series $\sum_{n, m} c_{n m} x_{n}^{*} \otimes x_{m}$ will be more simply denoted by $\sum_{j=1}^{\infty} b_{j} T_{j}$, where $b_{j}=c_{n m}$ in the appropriate ordering of the bases.

Let us also recall the following characterization of unconditional bases [5, p. 500] which is a quantitative version of Tutaj's observation [6] concerning the equivalence of unconditionality and the properties UL and UL*:

(*) $\quad A$ basis $\left\{x_{n}\right\}_{n=1}^{\infty}$ for $E$ is unconditional $\Leftrightarrow$ there are constants $\alpha$ and $\beta, 0<\alpha \leq 1 \leq \beta$, so that $\alpha\left\|\sum_{i=p}^{q}\left|a_{i}\right| x_{i}\right\| \leq\left\|\sum_{i=p}^{q} a_{i} x_{i}\right\| \leq$ $\beta\left\|\sum_{i=p}^{q}\left|a_{i}\right| x_{i}\right\|$ for all $1 \leq p \leq q<\infty$ and for all scalars $a_{p}, a_{p+1}, \ldots$ $\ldots, a_{q}$. 
Now, suppose $\left\{x_{n}, x_{n}^{*}\right\}_{n=1}^{\infty}$ is an unconditional basis for a reflexive Banach space $E$ and the series $\sum_{n, m}\left|a_{n m}\right| x_{n}^{*} \otimes x_{m}=\sum_{j=1}^{\infty}\left|b_{j}\right| T_{j}$ converges in the space $K(E)$ of compact operators on $E$. Then the sequence of partial sums of this series is Cauchy, so given any $\varepsilon>0$ there exists some $p_{0}$ for which $\| \sum_{j=p_{0}}^{q}\left|b_{j}\right| T_{j}||<\varepsilon$ for all $q \geq p_{0}$. An inspection of the ordering of the basis $\left\{x_{n}^{*} \otimes x_{m}\right\}$ shows that any such partial sum is of the form $\sum_{k \in A_{q}} x_{k}^{*} \otimes\left|v_{k}\right|$, where $A_{q}$ is a subset of $\left\{1, \ldots, N_{q}\right\}$ for some $N_{q}$, $v_{k}=\sum_{i \in \sigma_{k}} b_{i_{k}} x_{i}$ for $\sigma_{k}$ some subset of $\left\{1, \ldots, N_{k}\right\}$, and $\left|v_{k}\right|=\sum_{i \in \sigma_{k}}\left|b_{i_{k}}\right| x_{i}$ for each $k \in A_{q}$.

If $\left\|\sum_{j=p_{0}}^{q}\left|b_{j}\right| T_{j}\right\|<\varepsilon$ for some $p_{0}$ and some $q \geq p_{0}$, then by the above (and the definition of the norm in $K(E)$ ) we have

$$
\sup _{\|x\| \leq 1}\left\|\sum_{k \in A_{q}}\left\langle x_{k}^{*}, x\right\rangle\left|v_{k}\right|\right\|<\varepsilon .
$$

From the characterization $(*)$ of unconditional bases it follows that there are positive constants $\alpha$ and $\beta$ so that, for each $x=\sum_{n=1}^{\infty}\left\langle x_{n}^{*}, x\right\rangle x_{n}$ with $\|x\| \leq 1$, we have $\||x|\|=\left\|\sum_{n=1}^{\infty}\left|\left\langle x_{n}^{*}, x\right\rangle\right| x_{n} \mid\right\| \leq \alpha$, so

$$
\begin{aligned}
\varepsilon & >\left\|\sum_{j=p_{0}}^{q}\left|b_{j}\right| T_{j}\right\|=\left\|\sum_{k \in A_{q}} x_{k}^{*} \otimes\left|v_{k}\right|\right\| \\
& \geq \alpha \sup _{\|x\| \leq 1}\left\|\sum_{k \in A_{q}}\left\langle x_{k}^{*}, x\right\rangle\left|v_{k}\right|\right\| \\
& =\alpha \sup _{\|x\| \leq 1}\left\|\sum_{k \in A_{q}}\left\langle x_{k}^{*}, x\right\rangle \sum_{i \in \sigma_{k}}\left|b_{i_{k}}\right| x_{i}\right\| \\
& \left.\geq \frac{\alpha}{\beta} \sup _{\|x\| \leq 1}\left\|\sum_{k \in A_{q}}\left\langle x_{k}^{*}, x\right\rangle \sum_{i \in \sigma_{k}} b_{i_{k}} x_{i}\right\| \quad \text { (again, by }(*)\right) \\
& =\frac{\alpha}{\beta}\left\|\sum_{k \in A_{q}} x_{k}^{*} \otimes v_{k}\right\|=\frac{\alpha}{\beta}\left\|\sum_{j=p_{0}}^{q} b_{j} T_{j}\right\|,
\end{aligned}
$$

and so $\left\|\sum_{j=p_{0}}^{q} b_{j} T_{j}\right\|<(\beta / \alpha) \cdot \varepsilon$. That is, if $\sum_{j=1}^{\infty}\left|b_{j}\right| T_{j}$ converges then the sequence of partial sums of the series $\sum_{j=1}^{\infty} b_{j} T_{j}$ is Cauchy, hence convergent in $K(E)$, and the theorem is proved.

\section{References}

[1] B. R. Gelbaum and J. Gil de Lamadrid, Bases of tensor products of Banach spaces, Pacific J. Math. 11 (1961), 1281-1286.

[2] S. Karlin, Bases in Banach spaces, Duke Math. J. 15 (1948), 971-985.

[3] J. Lindenstrauss, On a certain subspace of $l^{1}$, Bull. Acad. Polon. Sci. Sér. Sci. Math. Astronom. Phys. 12 (1964), 539-542. 
[4] A. Pełczyński and S. Kwapień, The main triangle projection in matrix spaces and its applications, Studia Math. 34 (1970), 43-63.

[5] I. Singer, Bases in Banach Spaces I, Grundlehren Math. Wiss. 154, Springer, New York, 1970

[6] E. Tutaj, On Schauder bases which are unconditional-like, Bull. Polish Acad. Sci. Math. 32 (1985), 137-146.

[7] - Some observations concerning the classes of unconditional-like basic sequences, ibid. 35 (1987), 35-42.

Department of Mathematics

Virginia Polytechnic Institute and State University

Blacksburg, Virginia 24061-0123

U.S.A.

Reçu par la Rédaction le 17.1.1996 